\title{
Unrestricted vision
}

\section{Katy Schmidt}

Cell Microscopy Center, Department of Cell Biology, University Medical Center Utrecht, AZU, Room H02.313, Heidelberglaan 100, 3584 CX Utrecht, The Netherlands

Unrestricted vision can mean to see the full extent of things ahead and how they should turn out in the future but also of course, to have clear 20/20 eyesight. Let us take the latter as an example to explain what is meant by the former in this case. Vision is fascinatingly complex, and thus, it is not surprising that perturbation of eye sight can have many different underlying genetic causes, not to mention potential environmental and metabolic influences which are much more difficult to trace and generally polygenic in most cases. To date, a total of 214 genes have been linked to retinal disorders (http://www.sph.uth.tmc. edu/Retnet/), 49 of these, or nearly a quarter, is be causative for or involved in Retinitis Pigmentosa. Most prominently among them is rhodopsin in which around 120 distinct mutations have been identified [1]. To get to the point, different causes are likely to be covered by nearly as many distinct research areas; each of them will have its own array of specialist journals which more often than not can only offer restricted access to published articles with the result that interested scientists regularly have to ignore some publications when interpreting their own results. This is true for a lot of research topics but the visual pigment rhodopsin and Retinitis Pigmentosa are prime examples of this problem. The approaches to tackle this molecule and its diseasecausing mutations range from crystallography and chemistry across all aspects of biology to clinical and epidemiological studies. Practically no institution can cover all relevant journals with licences to grant access and therefore inevitably, a considerable number of articles will not be available for a lot of researchers although they might be of value.

To illustrate this issue in more detail and raise some questions alongside, we will detail some of the approaches and recent advances in the field. Rhodopsin is a canonical G-protein coupled receptor and consists of a protein part, opsin, and its covalently but reversibly bound chromophore, 11-cis retinal. When 11-cis retinal encounters a photon its conformation changes to all-trans retinal. As a consequence, the protein part undergoes several stages of conformational changes before all-trans retinal is released and a second messenger cascade is triggered eventually resulting in hyperpolarisation of the photoreceptor. This generates an action potential which will be integrated with signals from other photoreceptors and transmitted to the optical cortex. This superficial explanation of light perception is known since a long time and widely accepted textbook knowledge. Actually, there are still a lot of unknowns in the whole process and some very recent exciting developments. As a transmembrane protein rhodopsin is notoriously difficult to crystallise and although tremendous progress has been made over the past decade since the first structure has been published, the various activation states and conformational changes in their chronological sequence are still not fully understood. Just a month ago, a study combining NMR spectroscopy and computational simulations suggested that the starting point of the photocascade might even less clear cut than previously thought because it may be driven by a cluster of activated conformations of rhodopsin [2] rather than one specific state. This finding could be re-inforced by and certainly should be seen in conjunction with a different study investigating the interaction between rhodopsin and its G-protein, transducin [3]. Retinal and transducin roughly bind in the same patch of rhodopsin and this work suggested that the interaction between both proteins could be more dynamic than assumed so far and may actually occur in the dark already. This could mean that the $3 \mathrm{D}$ confirmation of rhodopsin in the cytoplasmic part could be much more flexible than the general assumption was up to date. Both studies together could have big implications in the search of drugs modulating activation and downstream signal transduction of rhodopsin [4]; but since at least the structural article is not open access, one can just hope that it will be available to all drug-developing groups.

The interaction with transducin, the compulsory triple G-protein of rhodopsin is not only of biochemical and crystallographic interest, this is also where human genetics come into play. Rhodopsin is a the heart of the visual (or retinoid) cycle of vertebrate animals (for more detail see [5]). The main purpose of the visual cycle is to re-isomerise 11-cis retinal and transport it back to photoreceptors for re-use. This involves quite a few enzymes and binding proteins in the intercellular space, or in other words two different cell types, the retinal pigment epithelium and photoreceptors and the transport of retinal between the two by the interphotoreceptor retinoid binding protein (IRBP). Remarkably, mutations of nearly all of these enzymes and transport and binding proteins have been linked to Retinitis Pigmentosa just as rhodopsin but mutations in all three subunits of transducin (GNAT 1 to 3 ) have not. Like some point mutations of rhodopsin itself, GNAT1 and 2 can cause congenital stationary night blindness instead ([1] and http:// www.sph.uth.tmc.edu/Retnet/disease.htm\#03.102d and .htm\#01.103d, respectively). This could be informative for studying protein-protein interactions but also for drug development and therapeutic approaches. It is likely that this problem has already been included in some studies but no publication to this effect was found (or was accessible). On the other hand, the link between Retinitis Pigmentosa and two enzymes clearing photoreceptors from all-trans-retinal could now be explained better by a free-fulltext publication [6]. It has been shown that if retinal accumulates in photoreceptors, photoreceptors undergo apoptosis as they would in Retinitis Pigmentosa. It is still not completely understood what the mechanism of toxicity is but plasma membrane permeability and mitochondrial poisoning seem to be involved. It would be interesting here whether these new findings can be reproduced with patient samples or in animal models of Retinitis Pigmentosa and whether this could open up a new unexplored therapeutic possibility. Chances are these questions will be investigated because thanks to open access everyone interested will be able to get hold of the data.

*Corresponding author: Dr. Katy Schmidt, Cell Microscopy Center, Department of Cell Biology, University Medical Center Utrecht, AZU, Room H02.313, Heidelberglaan 100, 3584 CX Utrecht, The Netherlands, Tel: +31 887557653; Fax: +31 302541797; E-mail: k.schmidt@umcutrecht.nl

Received September 16, 2011; Accepted September 18, 2011; Published September 20, 2011

Citation: Schmidt K (2011) Unrestricted vision. Biochem \& Anal Biochem 1:102e doi:10.4172/2161-1009.1000102e

Copyright: (C) 2011 Schmidt K. This is an open-access article distributed under the terms of the Creative Commons Attribution License, which permits unrestricted use, distribution, and reproduction in any medium, provided the original author and source are credited. 
No-one can doubt how important eye-sight and vision is for human beings; we all are very visual animals after all. Being able to see with one's eyes and one's mind is invaluable and by and large the foundation of science. The need to put some more effort into researching this area will grow quite quickly in the near future. Perturbed eye-sight and blindness are predicted to increase rapidly with an aging population in industrialised nations [7]. Already in 2006 it has been estimated that world-wide 259 million people are visually impaired including blind [8] The enormous interest in vision is illustrated by the fact that more than 370 articles on rhodopsin were published as cited in PubMed in the past year. However, only about 80 of these were linked to free full articles by September 2011. Obviously one can rely on institutional licences to gain access to some recent publications but overall this poses a problem for asking crucial, important questions to advance science that have not already been answered elsewhere and more so, for subsequently putting results in context to other research and generating an informed and valid interpretation of data. How does one assess the significance and meaning of own research with regards to new - and older - discoveries in the field if it is not possible to access all of them? Even more crucial, how can results be interpreted in the context of research areas slightly outside of one's own field (which are less likely to be covered by institutional licences)? If pace and advancement in science are to be kept high than all researchers should access all information they need rather than being slowed down and limited to a subset of publications. The most obvious solution and wish for the future is open access journals with reasonable page charges (and funding bodies granting enough money for publications) so that scientists are able to give full access the preference. Additionally, any other media publishers can employ to draw attention to new scientific findings that might be of interest would be beneficial. Ideally, some journals should also be somewhat flexible with their scope so that more than just one aspect of a research topic can be included and everyone is encouraged to look beyond the own horizon. After all, choosing to become a scientist has something to do being curious, having an ideal and having vision, unrestricted vision.

\section{References}

1. Mendes HF, van der Spuy J, Chapple JP, Cheetham ME (2005) Mechanisms of cell death in rhodopsin retinitis pigmentosa: implications for therapy. Trends Mol Med 11: 177-185.

2. Mertz B, Struts AV, Feller SE, Brown MF (2011) Molecular simulations and solid-state NMR investigate dynamical structure in rhodopsin activation. Biochim Biophys Acta.

3. Dell'orco D, Koch KW (2011) A dynamic scaffolding mechanism for rhodopsin and transducin interaction in vertebrate vision. Biochem $\mathrm{J}$.

4. Yanamala N, Gardner E, Riciutti A, Klein-Seetharaman J (2011) The Cytoplasmic Rhodopsin-Protein Interface: Potential for Drug Discovery. Curr Drug Targets.

5. Kiser PD, Golczak M, Maeda A, Palczewski K (2011) Key enzymes of the retinoid (visual) cycle in vertebrate retina. Biochim Biophys Acta.

6. Maeda A, Maeda T, Golczak M, Chou S, Desai A, et al. (2009) Involvement of all-trans-retinal in acute light-induced retinopathy of mice. J Biol Chem 284 : 15173-15183.

7. Congdon N, O'Colmain B, Klaver CC, Klein R, Munoz B, et al. (2004) Causes and prevalence of visual impairment among adults in the United States. Arch Ophthalmol 122: 477-485.

8. Dandona L, Dandona $\mathrm{R}$ (2006) What is the global burden of visual impairment? BMC Med 4: 6 . 